\title{
Retracción de pastas de cemento en estado plástico y en la primera etapa de endurecimiento
}

\author{
MAURICIO OSSA, M.* - CLAUDIO MARCANTONINI, M.** - FAVIO COFRE, H.**
}

\begin{abstract}
$R E S U M E N$
Existen muchos datos sobre la retracción que tiene lugar en las pastas, morteros y hormigones a partir de las veinticuatro horas de su amasado; sin embargo, pocos datos existen de los cambios volumétricos que ocurren por debajo de este tiempo y esto es debido principalmente a las dificultades que se presentan en las medidas de estas variaciones volumétricas.

Los autores de este trabajo experimental se han ocupado de las medidas de los cambios de volumen que tienen lugar en las pastas de cemento a corto plazo, es decir, en tiempos por debajo de las veinticuatro horas, aunque en sus medidas hayan llegado hasta los tres días.

Los ensayos los han llevado a efecto sobre microprobetas de $10 \times 10 \times 40 \mathrm{~mm}$ fabricadas mediante prensado y conservadas durante el tiempo de las medidas en un ambiente isotérmico y no agresivo. Los estudios se han realizado sobre cuatro tipos de cementos compuestos por clinkeres de diferentes composiciones y adiciones de puzolanas o escorias de horno alto.
\end{abstract}

\author{
$S U M M A R Y$ \\ A great deal of information exists on the retraction \\ occurring in pastes, mortars and concrete, twenty-four \\ hours after being mixed; we have very little information, \\ however, concerning the volumetric changes which \\ take place in a shorter period of time, this being \\ mainly due to the difficulties which arise in measuring \\ these volumetric variations.
}

The authors of this experimental work have been concerned whith measuring the short-term changes in volume which take place in cement pastes, that is to say, in periods of less than twenty-four hours, although they have taken measurements for periods of up to three days.

Experiments have been carried out on microsamples of $10 \times 10 \times 40 \mathrm{~mm}$ manufactured using the pressing method and kept throughout the measuring period in an isothermic non-aggressive atmosphere. Research has been carried out on four types of cement made up of clinkers of different composition and admixtures of pozzolana or scoria for blast furnaces.

\section{INTRODUCCION}

La pasta de cemento experimenta variaciones volumétricas debido a modificaciones físicas y quimicas que ocurren en su interior y que se manifiestan especialmente durante su estado plástico y en menor grado durante su endurecimiento.

Esas variaciones de volumen son bastante complejas y no muy conocidas en las horas siguientes a la mezcla. Las investigaciones publicadas sobre retracción se refieren, en mayoria a la retracción de secamiento a largo plazo medida en probetas endurecidas, tanto de hormigón como de mortero, en las que el origen de longitud se tomó generalmente después de las 24 horas $(1,2,3$, $4,5)$.

\footnotetext{
* Investigador del IDIEM.

** Ingenieros Civiles, U. de Chile.
} 
Para las primeras horas, los estudios experimentales son más reducidos, debido principalmente a las dificultades de medición. Esos trabajos $(3,6,7,8$ y 9) han tratado sobre la retracción plástica (fig. 1) y la diferencia en sus efectos con la sedimentación. Ultimamente se ha distinguido un periodo de hinchamiento seguido de una segunda retracción $(1,10,11)$ (fig. 2).

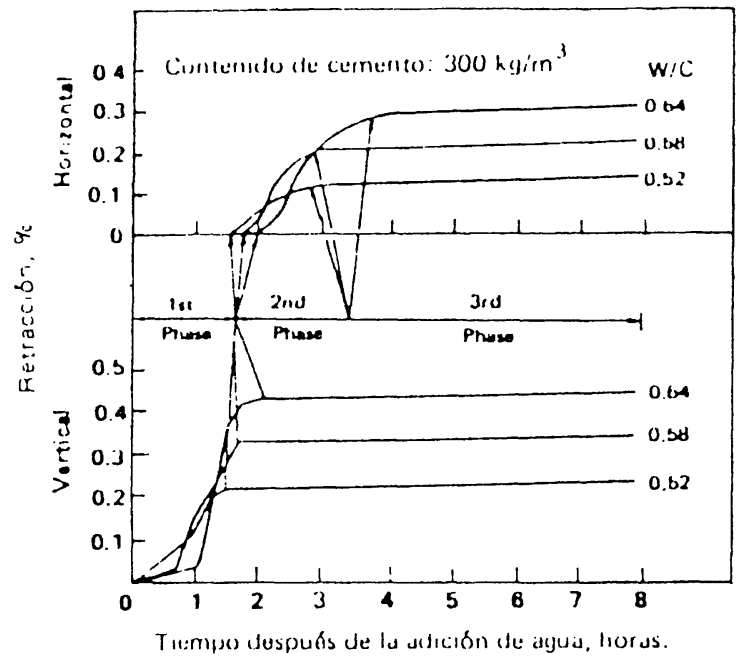

Fig. 1.-Efecto de la razón agua/cemento sobre las retracciones vertical y horizontal del hormigón. En un prisma de $10 \mathrm{~cm}$ de altura, la retracción vertical oscila entre 0,31 y $0,78 \%$, antes de que comience la retracción horizontal.

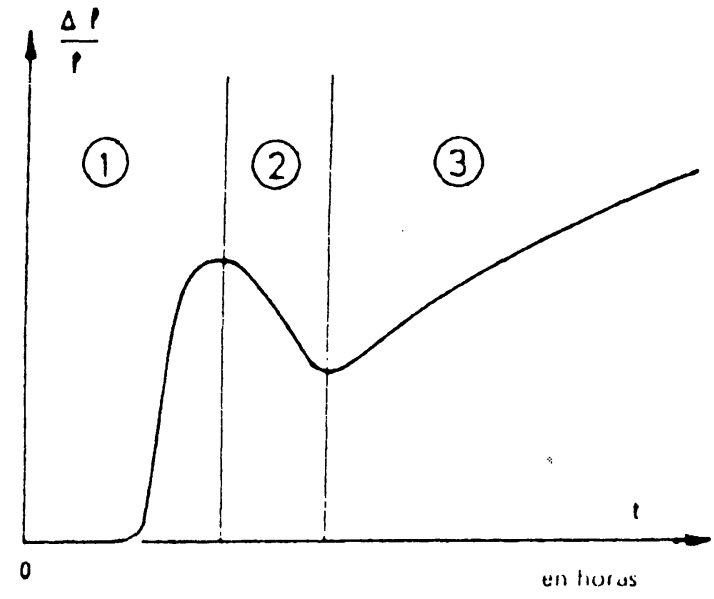

Fig. 2.-Los tres periodos de la deformación de una pasta normal:

1. Primera retracción.

2. Hinchamiento.

3. Segunda retracción.

No es fácil precisar el momento en que termina alguno de estos cambios volumétricos y comienza el siguiente. En algunos casos es detectable por cambios de pendiente o puntos de inflexión en la curva de retracción-tiempo; en otros, la diferencia es tan tenue que no alcanza a apreciarse.

En este estudio experimental, se consideraron variaciones de volumen debidas a la desecación y al proceso de hidratación de la pasta de cemento, las que se midieron en un ambiente isotermal y no agresivo.

\section{SEDIMENTACION}

Tanto en el mortero como en el hormigón, los fragmentos de árido se encuentran dispersos en la pasta cementante. Análogamente ocurre con la pasta de cemento; sus partículas forman con el agua una suspensión inestable, las que tienden a depositarse por acción de la gravedad. Como consecuencia de esto, se produce un descenso de la fase sólida y por ende, un acortamiento vertical, que en el caso del hormigón fresco, perjudica la homogeneidad en el interior. Con respecto a la fase liquida, el agua que sube puede formar canales de escurrimiento pobres en aglomerante $(12,13)$ lo que trae como consecuencia la falta de adherencia entre las partículas y un aumento de la relación agua-cemento en la superficie.

La sedimentación es tanto mayor: cuanto mayor es la profundidad del hormigón en estado plástico, cuanto mayor es el tiempo de inicio de fraguado y cuanto menos estable es la suspensión, lo que ocurrira con menos cemento, más agua, cemento más grueso y arena con menos finos $(1,14)$.

En obras de hormigón armado, las armaduras pueden impedir la sedimentación, originando fisuras entre el hormigón que queda suspendido y el que puede sedimentar libremente (12), produciendo un despegue del hormigón bajo las armaduras. 


\section{PRIMERA RETRACCION}

La primera retracción ha sido llamada también retracción plástica porque ocurre en el período plástico de la pasta de cemento, durante el fraguado y es causada principalmente por:

a) Pérdida de agua por evaporación, y

b) Combinación química de ella en el proceso de hidratación.

a) Retracción por evaporación. La pasta de cemento, conjunto rico en partículas finas, con una relación a/c de magnitud media, junto con una subdivisión extraordinariamente fina de sus espacios porosos ocasiona, durante la desecación y humidificación, variaciones volumétricas que pueden presentarse con magnitudes diferentes.

Por ejemplo, las condiciones de desecación dependen de la humedad relativa ambiente, de la velocidad del viento, de la temperatura, de la constitución de la pasta de cemento (ésta a su vez depende de la relación a/c, del grado de hidratación, del tipo de cemento) y de la influencia del anhídrido carbónico del aire $(12,13)$.

El mecanismo de la retracción puede explicarse sencillamente porque la pasta de cemento, con una gran subdivisión de sus espacios porosos y partículas muy finas de cemento, al tender a secarse, produce una migración del agua de la pasta a la superficie, provocando en los capilares que ocupaba fuerzas de tracción que tienden a acercar entre si las paredes de estos capilares. A su vez, estas fuerzas de retracción dependen del diámetro de los capilares. Mientras más pequeño es este diámetro, mayor es el grado de subdivisión del agua capilar y mayor es la fuerza de retracción.

b) Retracción por combinación química del agua. Al considerar la constitución física de los productos de hidratación del cemento se sabe (12) que el agua sufre una disminución de volumen por efecto de su combinación química. El volumen propio del material base, cemento más agua, es por lo tanto mayor que el que corresponde al producto hidratado. El grado de esta concentración volumétrica alcanza a ser aproximadamente un $25 \%$ en relación con el agua químicamente combinada.

Mediciones volumétricas hechas sobre probetas de pasta de cemento mediante el empleo de un dispositivo llamado volumenómetro (fig. 3), determinaron variaciones de volumen a lo largo del tiempo, producto del agua químicamente combinada, las que se muestran en la fig. 4. Dada la forma del aparato, estas medidas no incluyeron las variaciones producidas por la pérdida del agua hacia la atmósfera (12).

La velocidad del proceso de hidratación aumenta con mayor relación agua-cemento, provocando valores de retracción más altos para pastas de igual edad, confeccionadas con un mismo cemento (fig. 5). Relaciones agua-cemento inferiores a 0,25 pueden producir una hidratación incompleta de la pasta de cemento.

Según la información recopilada por J. Baron (11), la primera retracción comienza entre $0,5 \mathrm{y}$ 1,0 horas después de finalizado el mezclado y, en general, dura de 1,0 a 6,0 horas. Durante esta última etapa, aumenta linealmente en función del tiempo y luego se estabiliza bruscamente. Su valor máximo es muy elevado y según las condiciones ambientales varia entre 1,0 y $20,0 \cdot 10^{3}$. El autor confirma además la influencia preponderante sobre la primera retracción de la temperatura, la higrometria, el viento, la dosis de agua y de cemento y, también, de la autodesecación debida al proceso de hidratación. Esta última, se demuestra en la fig. 6, donde la retracción se ha manifestado también en la profundidad de una probeta moldeada. 


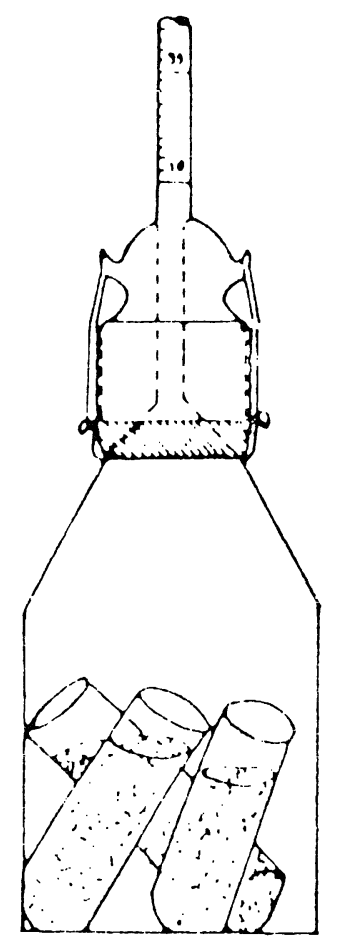

Fig. 3.-Volumenómetro. Dispositivo para medir variaciones de volumen de probetas de cemento.

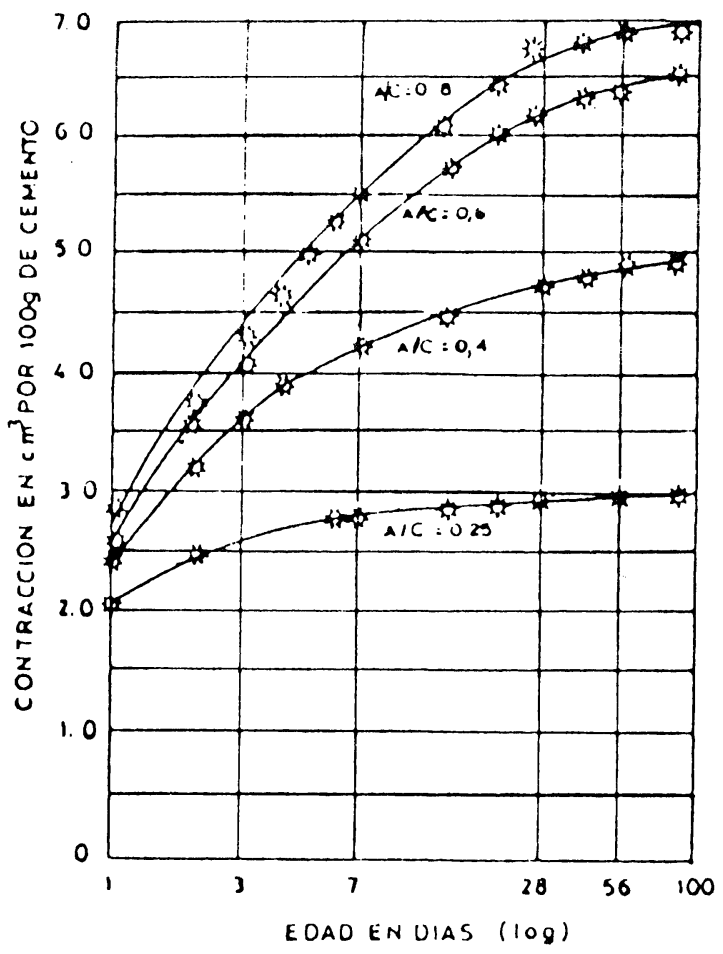

Fig. 5.-Curva de retraccion para distintas relaciones de agua-cemento.

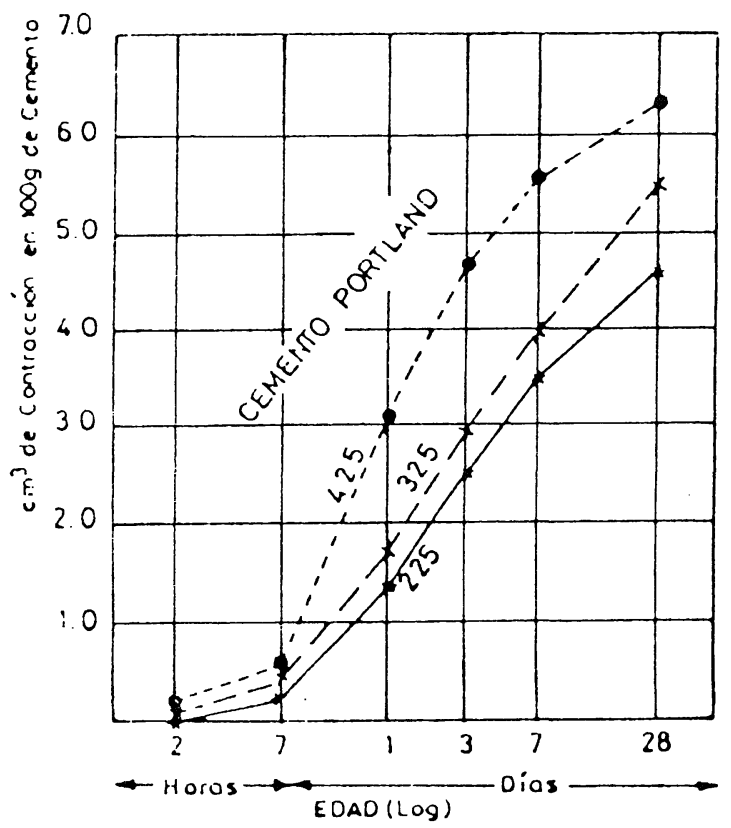

Fig. 4.-Curvas de retracción de cementos europeos, de distinta energia de endurecimiento a los 28 dias, en $\mathrm{kg} / \mathrm{cm}^{2}$.

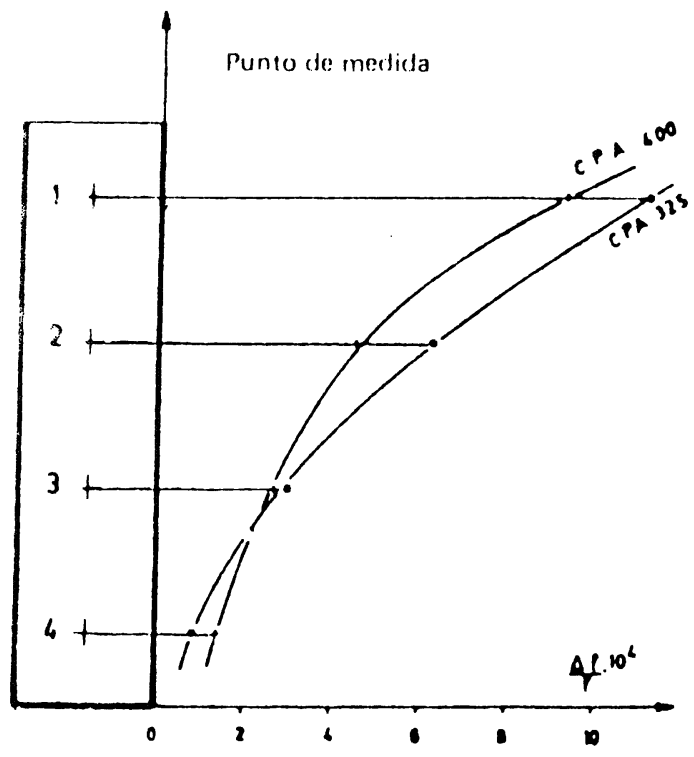

Fig. 6.-Variación de la primera retracción de una pasta normal en función de la profundidad en el molde. La probeta mide $7 \mathrm{~cm}$ de ancho, $28 \mathrm{~cm}$ de largo y $28 \mathrm{~cm}$ de profundidad. En la vecindad de la superficie expuesta (parte superior), la primera retracción es más fuerte, a causa de la evaporación de agua interna. Las deformaciones, cerca del fondo del molde, se reducen notablemente a consecuencia del roce.

CPA 400.-Alto contenido de $\mathrm{C}_{3} \mathrm{~A}$.

CPA 325.-Contenido medio de $\mathrm{C}_{3} \mathrm{~A}$. 


\section{HINCHAMIENTO}

El proceso de primera retracción está seguido generalmente por una expansión o hinchamiento de la pasta de cemento.

El hinchamiento es a veces muy diferente para distintos tipos de cemento y, más aún, con el mismo cemento no se han obtenido los mismos resultados. En algunos casos este fenómeno es tan leve que no alcanza a apreciarse en las medidas experimentales de longitud, que se realizan sobre la pasta.

El hinchamiento se debe a reacciones físicas y quimicas que ocurren durante la hidratación de la pasta de cemento. Es ciertamente un movimiento que acontece mientras la estructura se encuentra inestable y delicada (14).

Como posible causa de este hinchamiento puede ser la reacción del yeso que se agrega al clínker para regularizar la velocidad de hidratación del $\mathrm{C}_{3} \mathrm{~A}$. Un exceso de yeso provoca hinchamientos bastante rápidos, que se manifiestan en las primeras horas de medición y se debe a la formación del trisulfoaluminato cálcico hidratado o sal de Candlot (1).

La cal y la magnesia libres, al hidratarse, también producen aumentos de volúmenes, pero esas hidrataciones se realizan con lentitud y sus cambios volumétricos son apreciables después de varias semanas o meses.

El hinchamiento comienza entre 4 y 10 horas después del mezclado y se puede prolongar hasta 10 o más horas, siendo su magnitud del orden de $10^{-3}$.

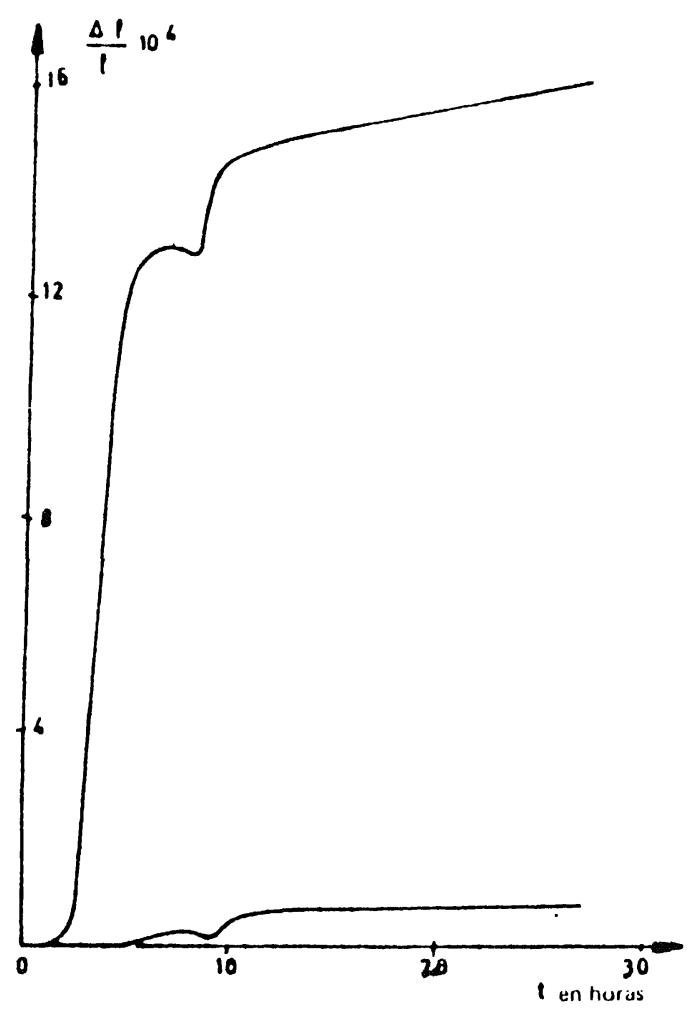

Fig. 7.-Mientras sobre la pasta la segunda retracción sobreviene bruscamente (curva superior), se puede constatar el mismo fenómeno sobre mortero (curva inferior). Estos resultados han sido obtenidos en condiciones isotérmicas.

\section{SEGUNDA RETRACCION}

La segunda retracción (o retracción de endurecimiento), se produce después del fraguado y a continuación del hinchamiento, con ausencia de evaporación y generada por modificaciones quimicas y fisicas durante la hidratación del cemento. Puede durar muchos días o varias semanas (2).

Según J. Baron (11), su duración puede ser de 2,0 horas a 10,0 horas y a veces llega a ser mayor. Su aparición puede presentarse de manera progresiva o instantánea. En este último caso, la curva de deformación de la probeta muestra un punto anguloso que se aprecia más claramente en pastas de cemento que en mortero (fig. 7). La velocidad de la segunda retracción pasa por un valor máximo y luego disminuye en forma constante. Una parte importante de la segunda retracción está directamente vinculada al fenómeno de hidratación del cemento y, por lo tanto, al igual que el periodo de hinchamiento, su desarrollo no se modifica aunque las probetas se encuentren expuestas o protegidas.

Más que los valores que alcanza la segunda retracción, interesa conocer la velocidad con que 
ella se produce, característica no mostrada por los ensayos tradicionales normalizados. E1 examen de fisuración de obras de hormigón y los resultados de ensayos de segunda retracción sobre los cementos correspondientes, permiten pensar que esta caracteristica del cemento es uno de los factores importantes en la formación de fisuras en las primeras horas o primeros dias siguientes a la colada del hormigón $(1,10,15)$, ya que la pasta de cemento no puede absorber la deformación por fluencia lenta $(1,12)$. Se piensa que el riesgo de fisuración precoz es más reducido cuando la velocidad máxima de segunda retracción es pequeña.

En los cementos siderúrgicos, el endurecimiento inicial es más lento. Debido a esto se necesita molerlos a finuras más elevadas que los cementos portland, y en general esto motiva en los primeros una mayor retracción por resecamiento.

De acuerdo a las teorías de Fernández París (16) las pastas hidratadas de cemento portland experimentan retracción, cuando el contenido de yeso es inferior al óptimo. Análogamente, cuando el contenido de yeso supera el óptimo, se producen expansiones debido a la formación de ettringita secundaria (formada posteriormente al periodo plástico).

\section{INFLUENCIA DE LA FORMA Y TAMAÑO DE LAS PROBETAS EN LAS MEDIDAS DE RETRACCION}

Se han hecho varias investigaciones sobre la influencia del tamaño y forma de las probetas, en las medidas de retracción $(17,18,19,20,21,22)$.

L'Hermite y Mamillan (17) midieron retracciones superficiales y axiles en probetas prismáticas homotéticas de un mismo hormigón, con tamaños desde $7 \times 7 \times 28 \mathrm{~cm}$ hasta $100 \times 100 \times 400 \mathrm{~cm}$ durante un periodo de 850 dias. De sus resultados obtenidos concluyeron que la retracción superficial se estabiliza aproximadamente a los 850 días, mientras que para esa edad la retracción axil del prisma de $100 \mathrm{~cm}$ de lado era la tercera parte de la retracción superficial y continuaba en evolución.

También compararon la retracción superficial y axil de probetas de igual razón (volumen/superficie) para placas y prismas concluyendo que este parámetro no era significativo y que de haber una relación, ella debería incluir la temperatura interior de la probeta y su espesor.

D. W. Hobbs (22) hizo medidas de retracción en probetas prismáticas de un mismo hormigón durante un periodo de 2.000 días. De la información obtenida, concluyó que la retracción a temprana edad disminuye con el aumento del tamaño de la probeta. Esta diferencia proporcional disminuye con el tiempo hasta hacerse aproximadamente independiente alrededor de los 3 años, y el monto de esta retracción está dado aproximadamente por una función:

$$
S=\mathrm{f}(A / V)^{\mathrm{n}} t
$$

donde: $A$ es el área de exposición, $V$ es el volumen, $n$ exponente que varia entre 1 y 2 (dependiendo del cemento, humedad relativa, carbonatación, etc.) y $t$ el tiempo.

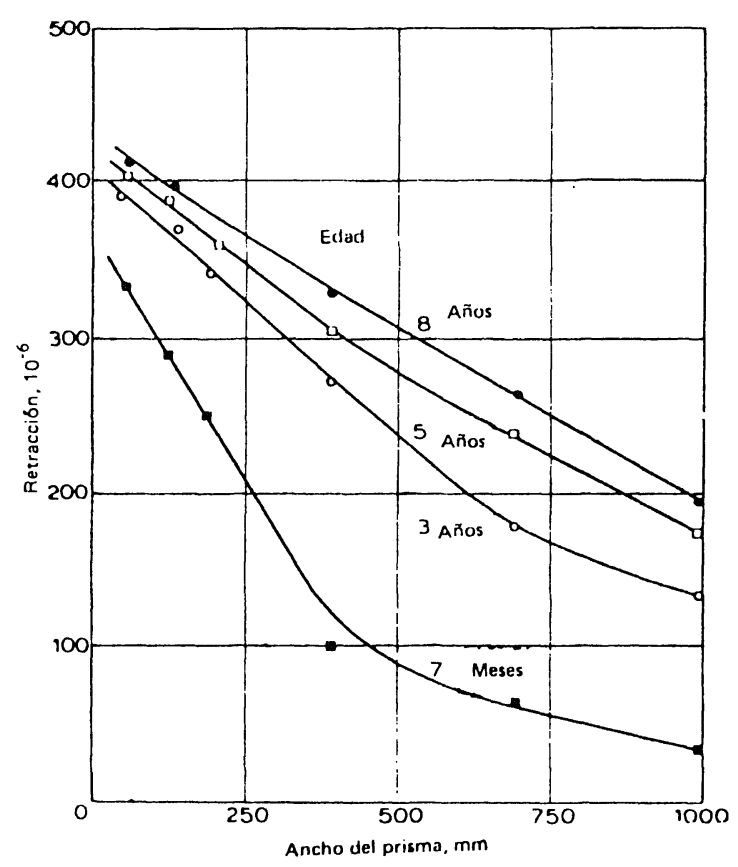

Fig. 8.- Relación entre retracción axil y ancho del prisma de hormigón, de sección cuadrada y razón longitud : ancho igual a 4 (con secamiento permitido en todas las superficies). 
A. Neville (23) señala la relación entre la retracción axil y el ancho de prisma de hormigón de razón longitud : ancho constante, como se observa en fig. 8.

J. Baron (11) demuestra el efecto reductor de retracción que produce en las primeras horas el roce de paredes de los moldes (fig. 9).

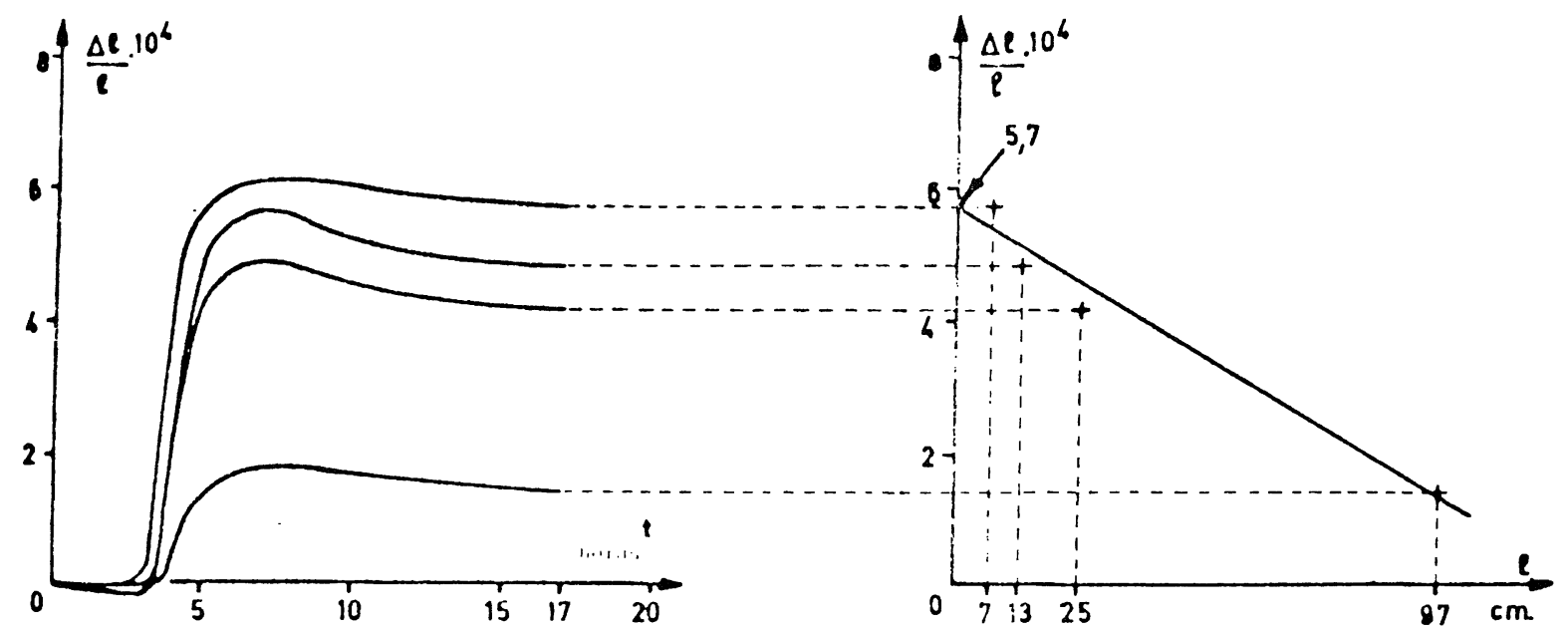

Fig. 9.-Influencia del roce sobre variaciones de longitud medidas.

A la izquierda aparecen representadas las variaciones de longitud de probetas de 7, 13, 25 y $97 \mathrm{~cm}$ de largo. Mientras más larga es la probeta, la variación relativa de longitud es más débil. Por extrapolación (a la derecha) se puede deducir una estimación del valor de retracción sin roce a las 17 horas: $5,7 \cdot 10^{-4}$. Se constata que (a la izquierda) más allá del primer máximo, las curvas son paralelas y que, en consecuencia, el roce no interviene.

Este resultado no es intuitivo: mientras menos larga es la probeta, menos interviene cl roce sobre el valor medido. En el limite, para una probeta de longitud nula, el roce, evidentemente, no interviene en nada.

\section{ENSAYOS DE RETRACCION DE CEMENTOS}

El objetivo de estos ensayos fue la medición de retracción a lo largo del tiempo, desde una hora después del amasado hasta una edad de 3 días de probetas de pasta de cementos, de grado corriente, con dimensiones de $10 \times 10 \times 40 \mathrm{~mm}$, y que se confeccionaron mediante una técnica especial, a partir de una relación agua : cemento de consistencia normal (Norma NCh 151. Of 68.).

\section{CEMENTOS EMPLEADOS}

Se seleccionaron cementos de grado corriente cuyas características se muestran en la Tabla I.

TABLA I

Composición y finura de los cementos

\begin{tabular}{|c|c|c|c|c|c|c|c|}
\hline \multirow{2}{*}{$\begin{array}{c}\text { Cemento } \\
\mathbf{N}^{\mathrm{o}}\end{array}$} & \multicolumn{4}{|c|}{ Clinker, \% } & \multicolumn{2}{|c|}{ Adición } & \multirow{2}{*}{$\begin{array}{l}\text { Finura } \\
\text { Blaine } \\
\mathrm{cm}^{2} / \mathrm{g}\end{array}$} \\
\hline & $\mathrm{C}_{3} \mathrm{~S}$ & $\mathrm{C}_{2} \mathrm{~S}$ & $\mathbf{C}_{\mathbf{3}} \mathbf{A}$ & $\mathrm{C}_{4} \mathrm{AF}$ & Tipo & $\%$ & \\
\hline 1 & 68 & 13 & 5 & 12 & Puzolana & 29 & 4.000 \\
\hline 2 & 61 & 13 & 10 & 10 & Puzolana & 30 & 4.200 \\
\hline 3 & 52 & 22 & 7 & 11 & Puzolana & 32 & 4.000 \\
\hline 4 & 60 & 19 & 8 & 7 & Escoria A.H. & 60 & 4.200 \\
\hline
\end{tabular}




\section{CONFECCION DE PROBETAS DE PASTA DE CEMENTO}

La pasta de cemento se obtuvo mezclando agua y cemento en una máquina mezcladora normal Tonindustrie durante $90 \mathrm{~s}$ a 140 r.p.m. y durante 90 s a 285 r.p.m. Finalmente la pasta preparada se tamizó a través de tamices DIN de $3,15 \mathrm{~mm}$ y $1,6 \mathrm{~mm}$ de abertura para separar los gruesos.

Las probetas se confeccionaron mediante un aparato diseñado y construido en el IDIEM para este trabajo. (Fig. 10). Consiste en un bloque de acero en 2 secciones: un cuerpo con 3 perforaciones rectangulares de $10 \times 40 \mathrm{~mm}$ y una perforación rectangular horizontal por la cual desliza una pieza móvil de $10 \mathrm{~mm}$ de espesor, con una perforación rectangular también de $10 \times 40 \mathrm{~mm}$ que sirve de molde y aloja finalmente a la probeta durante su confección.

La fig. 11 muestra el funcionamiento de este aparato. Se efectúa el llenado del molde probeta en posición horizontal con pasta de cemento por la parte superior de la perforación I, de manera que la columna inicial de pasta de cemento no sea mayor que $15 \mathrm{~mm}$ (probeta + residuo) (fig. 11.a), y se aplica luego la presión de moldeo a través del pistón mediante un sistema de palancas (fig. 12). Por el deslizamiento del molde o pieza móvil se cizalla la columna de pasta de cemento comprimida, quedando así la probeta alojada en el molde. Finalmente por medio de la perforación III se expulsa la probeta del molde.

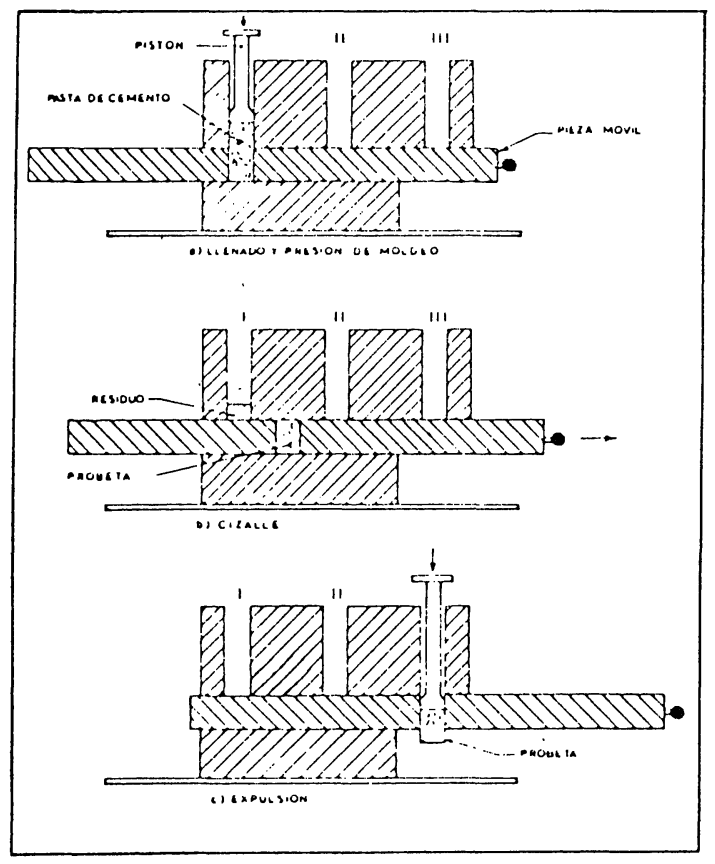

Fig. 11.-Esquema de funcionamiento del molde para probetas.

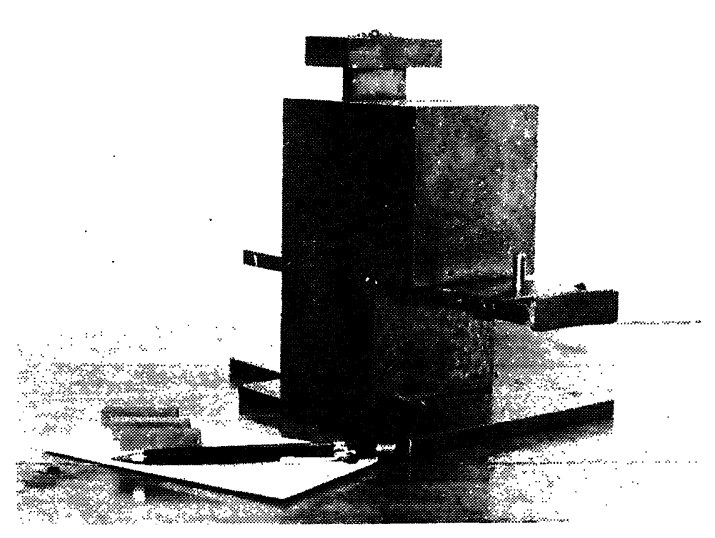

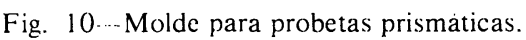

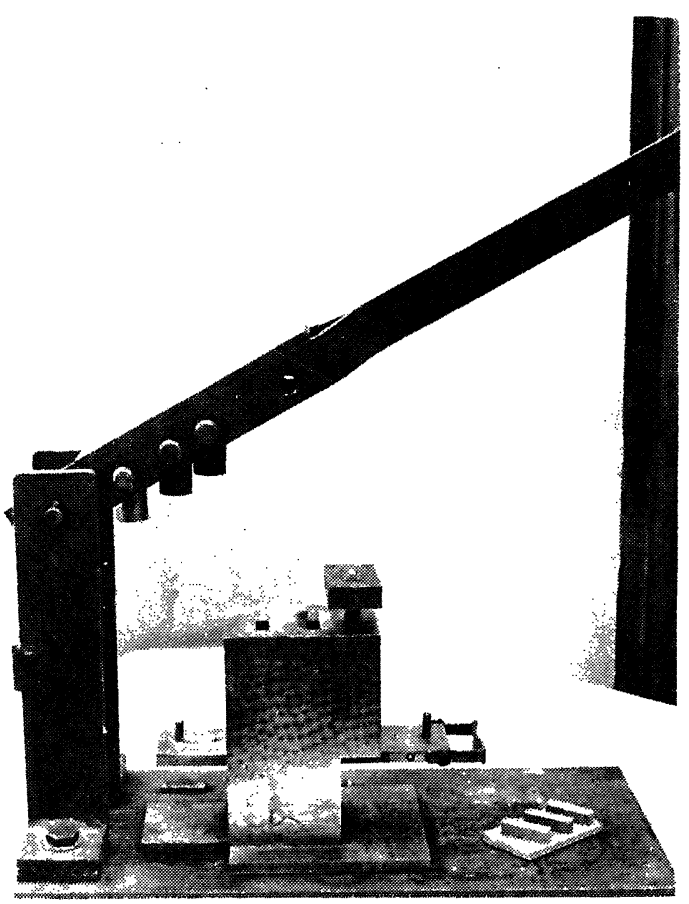

Fig. 12.-Sistema de palancas.

Para retirar el residuo se devuelve la pieza móvil a la posición inicial a), se empuja al interior del cuerpo móvil mediante el pistón y en la posición c) se expulsa del aparato. 


\section{CARACTERISTICAS FINALES DE LAS PROBETAS}

Las pastas de cemento preparadas con una consistencia normal, sufrían una expulsión del agua en exceso al ser comprimidas por la máquina de conformación, dejando a las probetas con las características que se indican:

- dimensiones: $\quad 40 \times 10 \times 10 \mathrm{~mm}$

- relación agua cemento final: $\mathrm{a} / \mathrm{c}=0,20$

- presión de moldeo recibida: $p=32 \mathrm{~kg} / \mathrm{cm}^{2}(3,14 \mathrm{MPa})$

- peso: $\quad w=8,9 \pm 0,05 \mathrm{~g}$

La determinación de la relación agua: cemento final se hizo por diferencia de peso entre probetas recién moldeadas e inmediatamente pulverizadas y secadas en horno para evaporar el agua remanente.

\section{METODO DE MEDICION DE LA RETRACCION}

Después de moldeadas las probetas se recogieron con porta-objetos de acrílico, quedando todas sus caras, excepto su base, expuestas al medio ambiente desecante del laboratorio que normalmente registra temperaturas de $21 \pm 1^{\circ} \mathrm{C}$ y humedades relativas de $54 \pm 2 \%$.

Se confeccionaron entre 3 y 6 probetas por cada tipo de cemento, a las que después de moldeadas, se les pegaba a cada una de sus caras extremas un tope de cobre mediante una resina de fraguado rápido. Este tope de medida consistió en un filamento de cobre de aproximadamente $0.5 \mathrm{~mm}$ de diámetro, dispuesto en forma de ele minúscula manuscrita $(l)$ y cuyos extremos eran pegados a la cara de la probeta (fig. 13).

Cada media hora se tomaron lecturas de los extremos de los topes mediante un microscopio micrométrico AMSLER (fig. 14). Este microscopio proporcionaba un desplazamiento horizontal del cuerpo óptico de 50 milímetros como máximo, recorriendo totalmente la probeta. El movi-

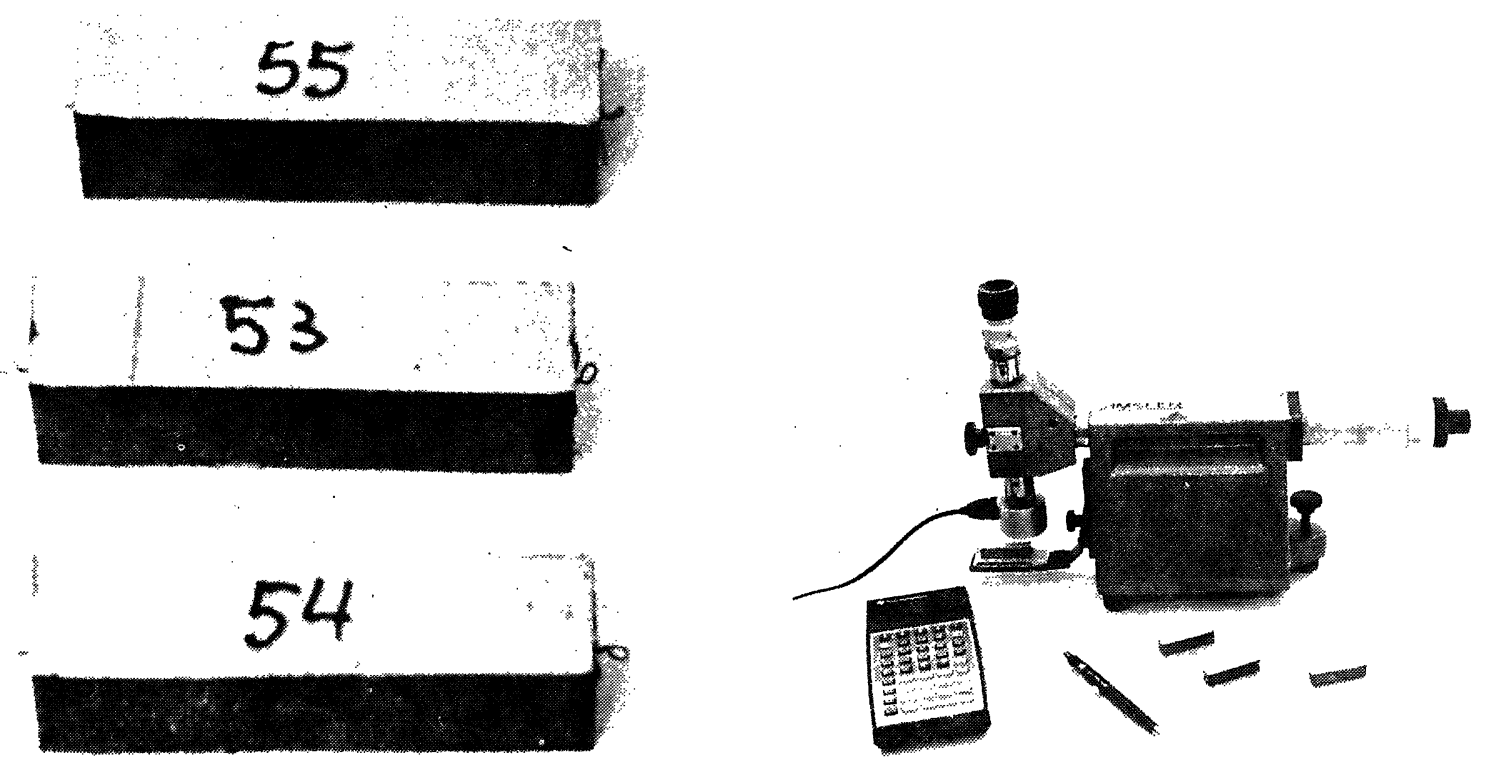

Fig. 13.- Probetas prismaticas de pasta de cemento con sus topes para medir retracción.

Fig. 14.-Microscopio micrometrico AMSLER. 
miento era producido por un tornillo sinfin conectado a un cilindro graduado que permite leer con una precisión de 2 milésimas de milimetro.

La longitud inicial de las probetas se midió una hora después de haberse confeccionado la pasta. Todas las medidas se obtuvieron por diferencia de lectura entre los topes.

La mayor parte de la información que ha podido recogerse sobre medidas experimentales de la primera retracción se refieren a probetas de pasta de cemento con tres o cinco de sus caras cubiertas por las paredes del molde. De esta manera la deformación medida en ella no fue de forma absoluta debido a la primera retracción, sino a una deformación resultante de retracción y de restricciones mecánicas de roce con las caras del molde.

Las probetas prismáticas de este trabajo confeccionadas por compactación no presentaron este inconveniente ya que todas sus caras, excepto su base recubierta con una capa de aceite, estaban libres y expuestas al medio ambiente.

\section{RESULTADOS OBTENIDOS}

Las deformaciones de retracción obtenidas en los ensayos se muestran desde la fig. 15 a la fig. 18 para los distintos cementos.

Las figs. 15 y 17 señalan las retracciones en función del tiempo de 6 probetas compañeras de una misma muestra con cementos $\mathrm{N}^{\text {os }} 1$ y 3 respectivamente, ambos de grado corriente. Análogamente en cada una de las figs. 16 y 18 aparecen las retracciones de 3 probetas de cemento $\mathrm{N}^{\circ} 2$ y de cemento $\mathrm{N} .^{\circ} 4$. En las figs. 19, 20, 21 y 22 se representan las curvas de retracción media de cada tipo de cemento. En todos estos gráficos se ha tomado como origen de tiempo el momento de amasado de las pastas de cemento.

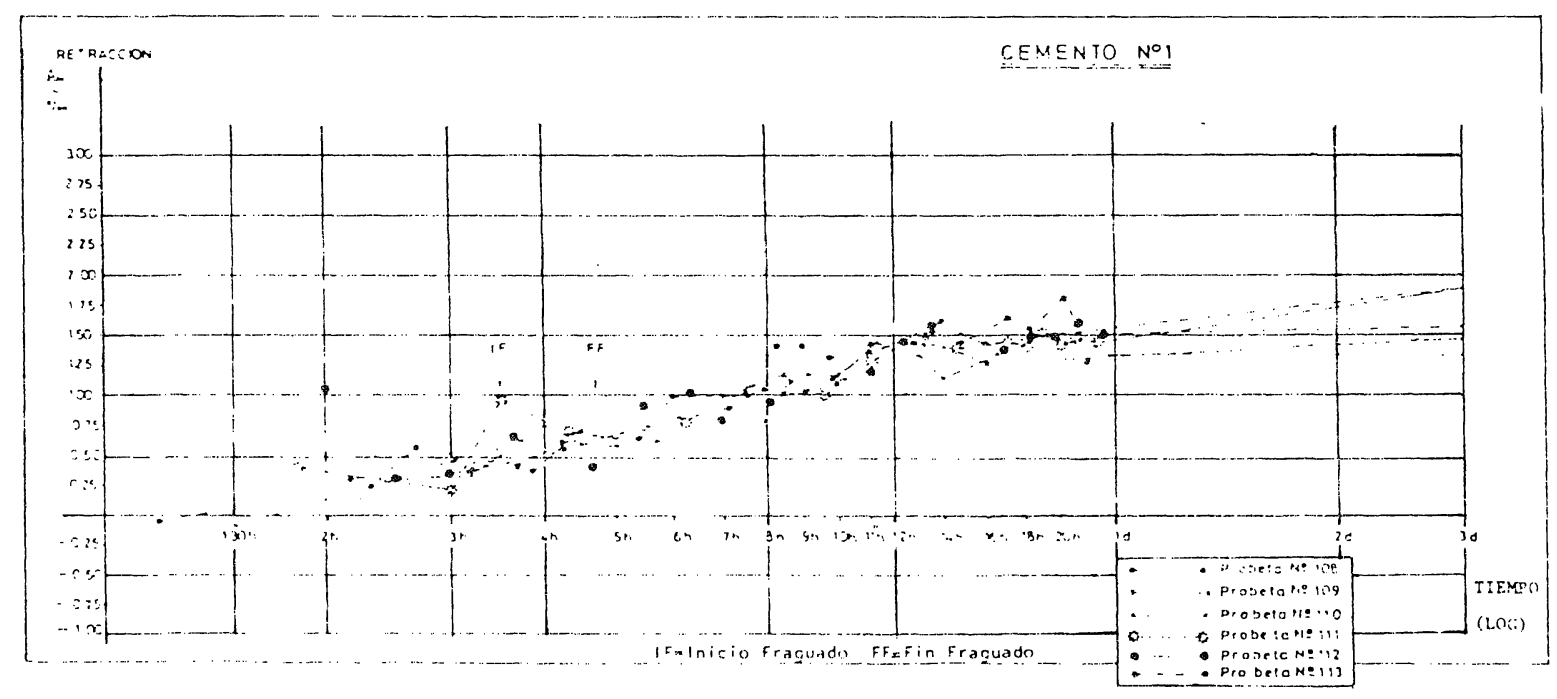

Fig. 15.--Relación retracción : edad obtenida de 6 miniprobetas de pasta del cemento N." 1 . 


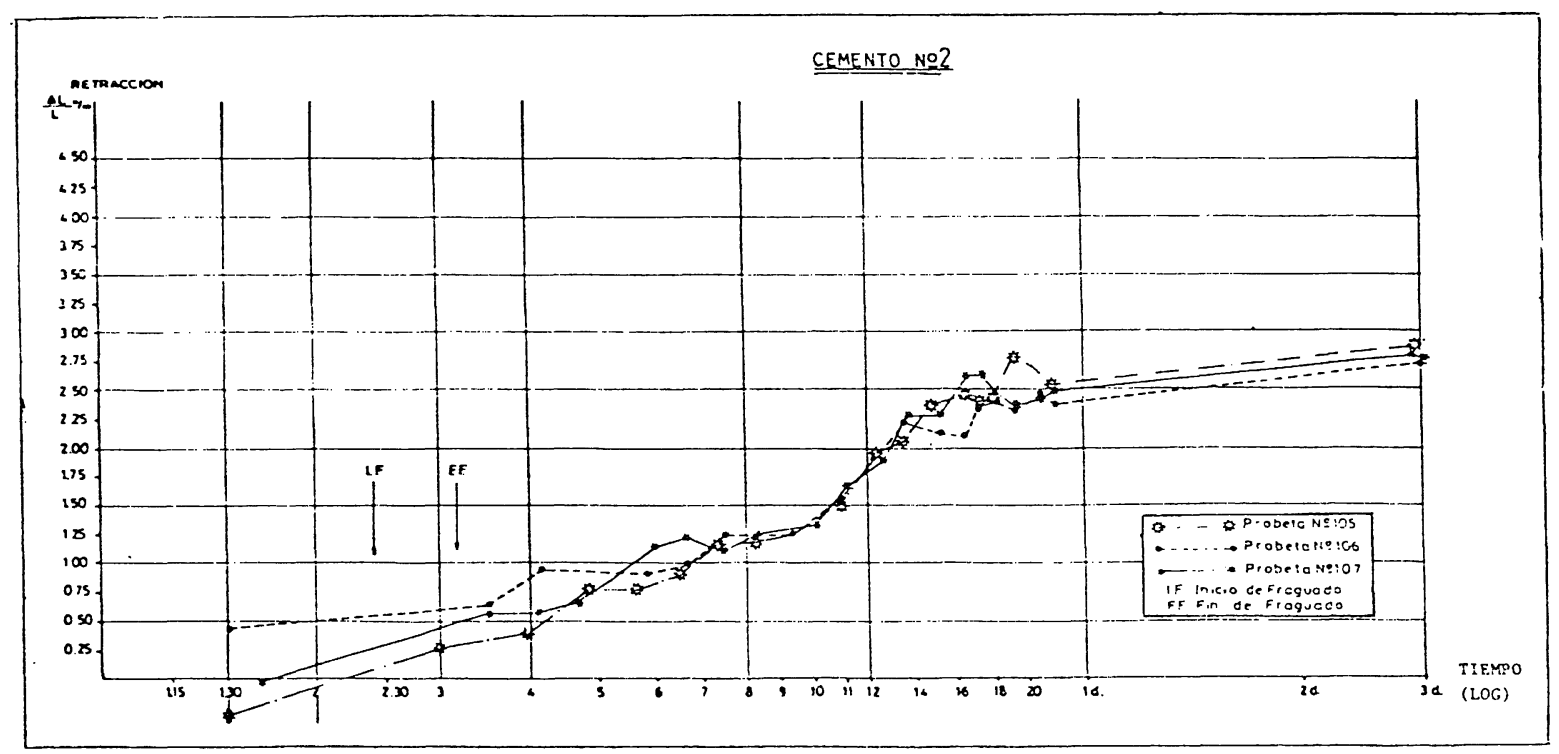

Fig. 16.-Relación retracción : edad obtenida de 3 miniprobetas de pasta del cemento $\mathrm{N}^{\circ} 2$.

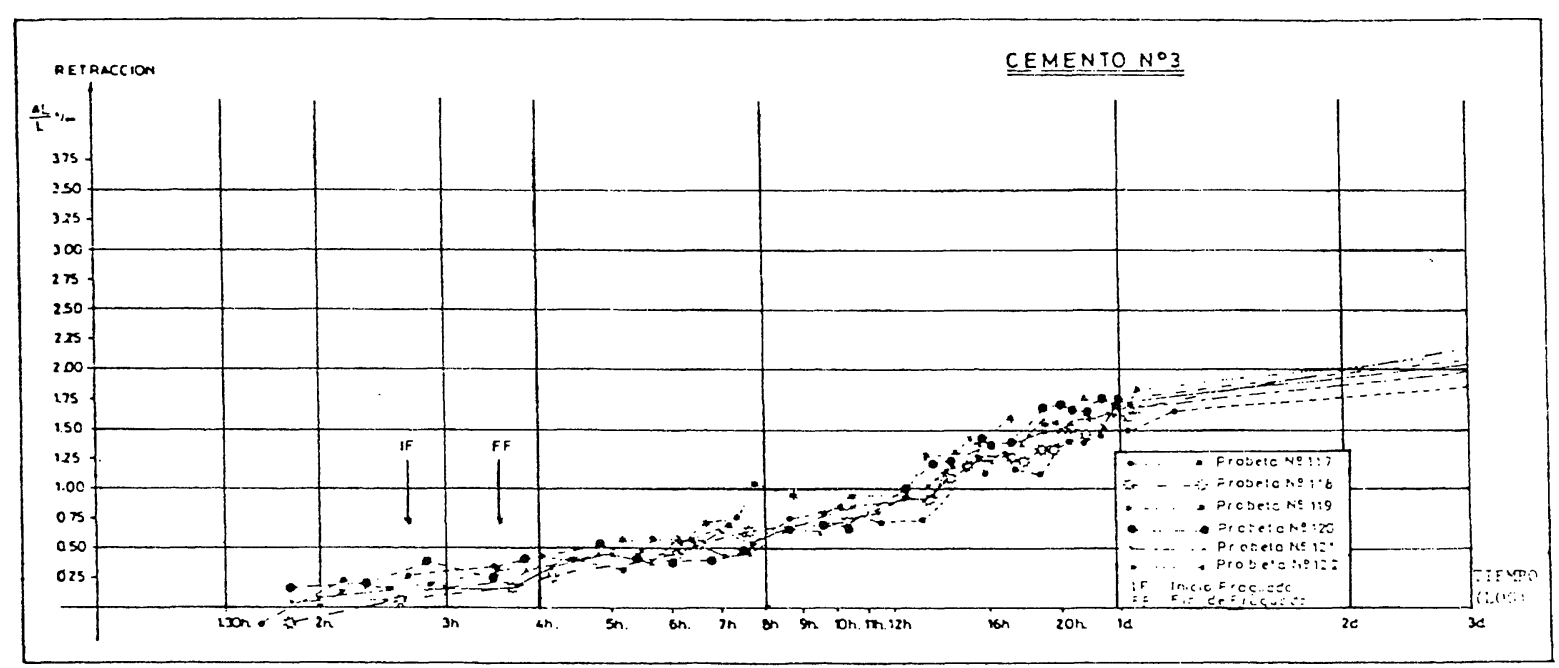

Fig. 17.- Relación retracción : edad obtenida de 6 miniprobetas de pasta del cemento $\mathrm{N}^{\circ}{ }^{\circ} 3$.

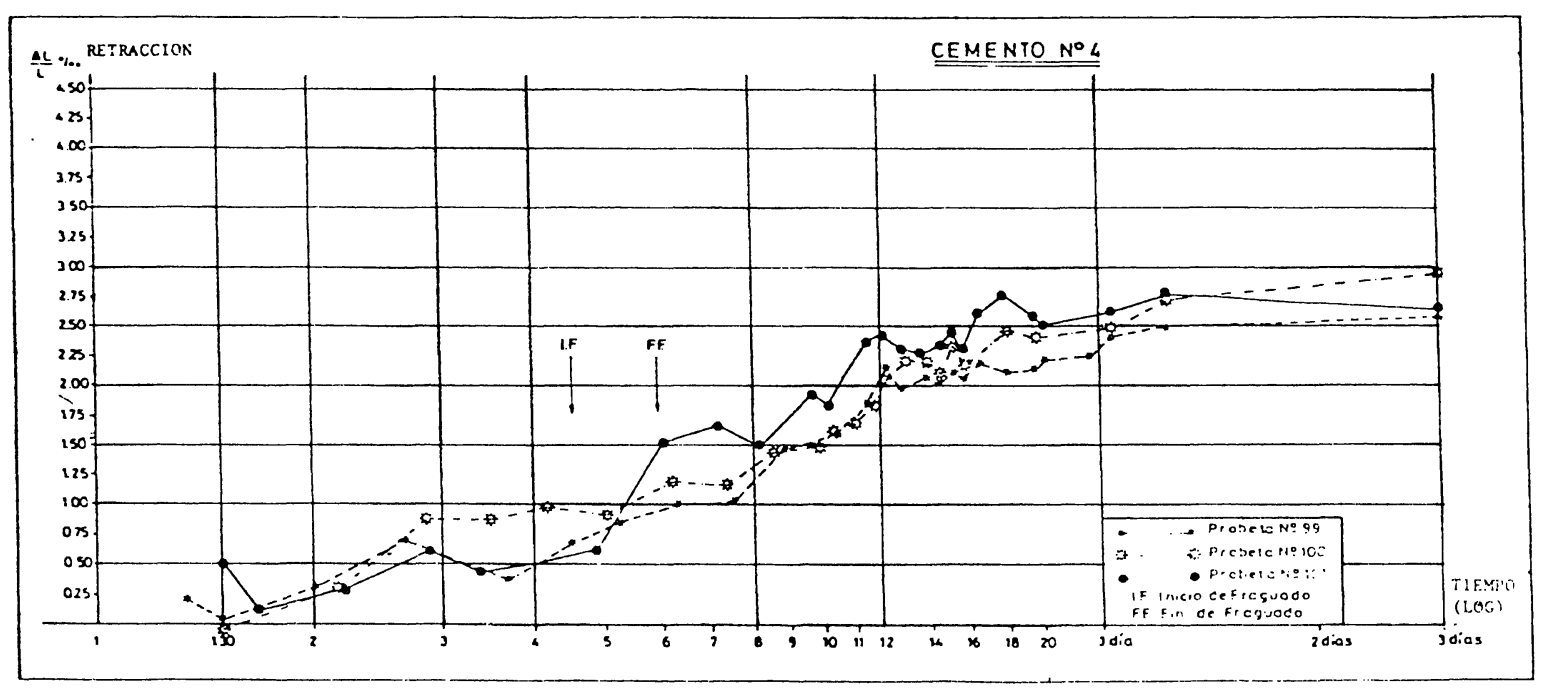

Fig. 18.- Relación retracción : edad obtenida de 3 miniprobetas de pasta del cemento N. ${ }^{\circ} 4$. 
RETRACCION

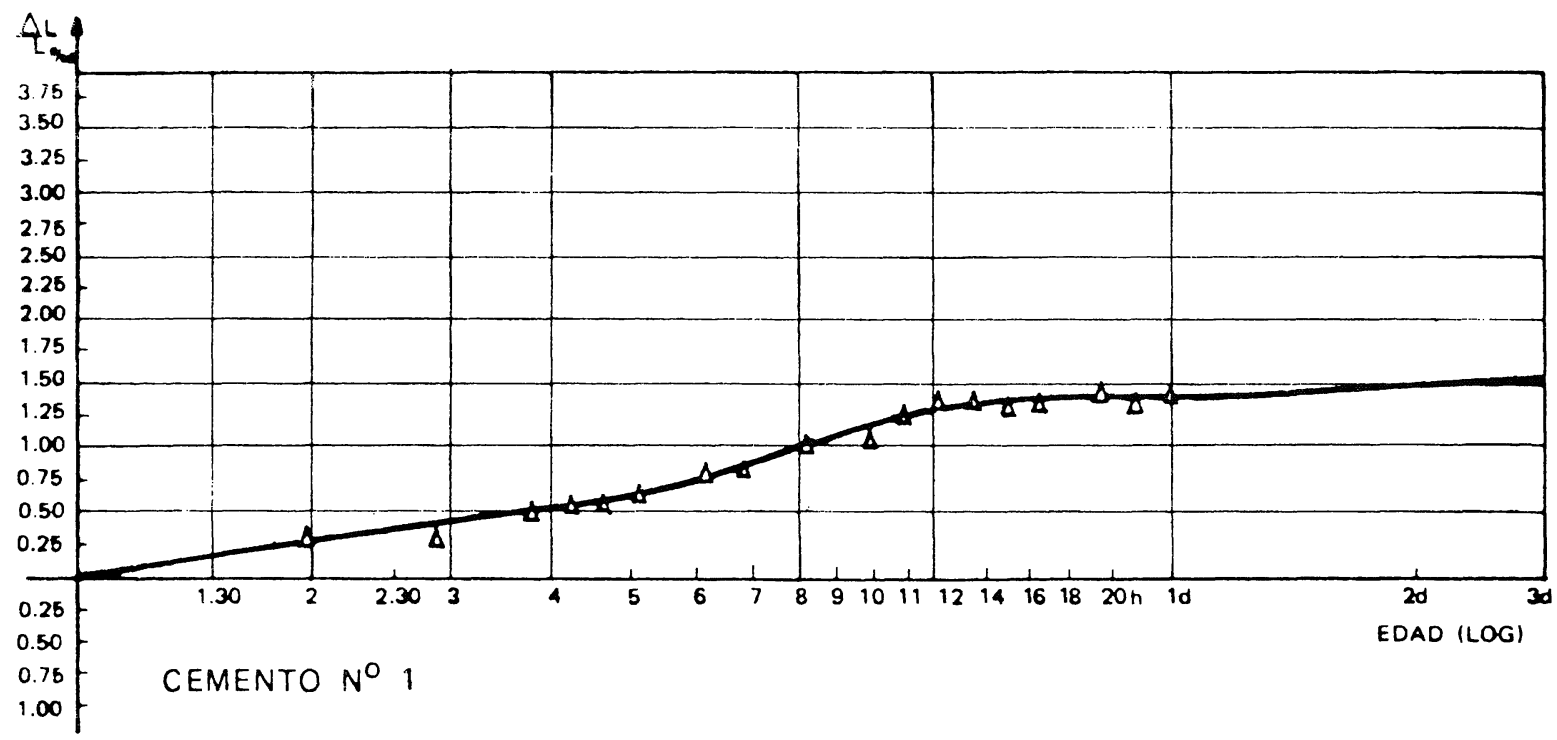

Fig. 19.--Promedio de retracciones medidas en miniprobetas de pasta del cemento $\mathrm{N}^{\circ}{ }^{\circ} 1$ en función de la edad. La curva ajustada permite medir la velocidad máxima de la segunda retracción.

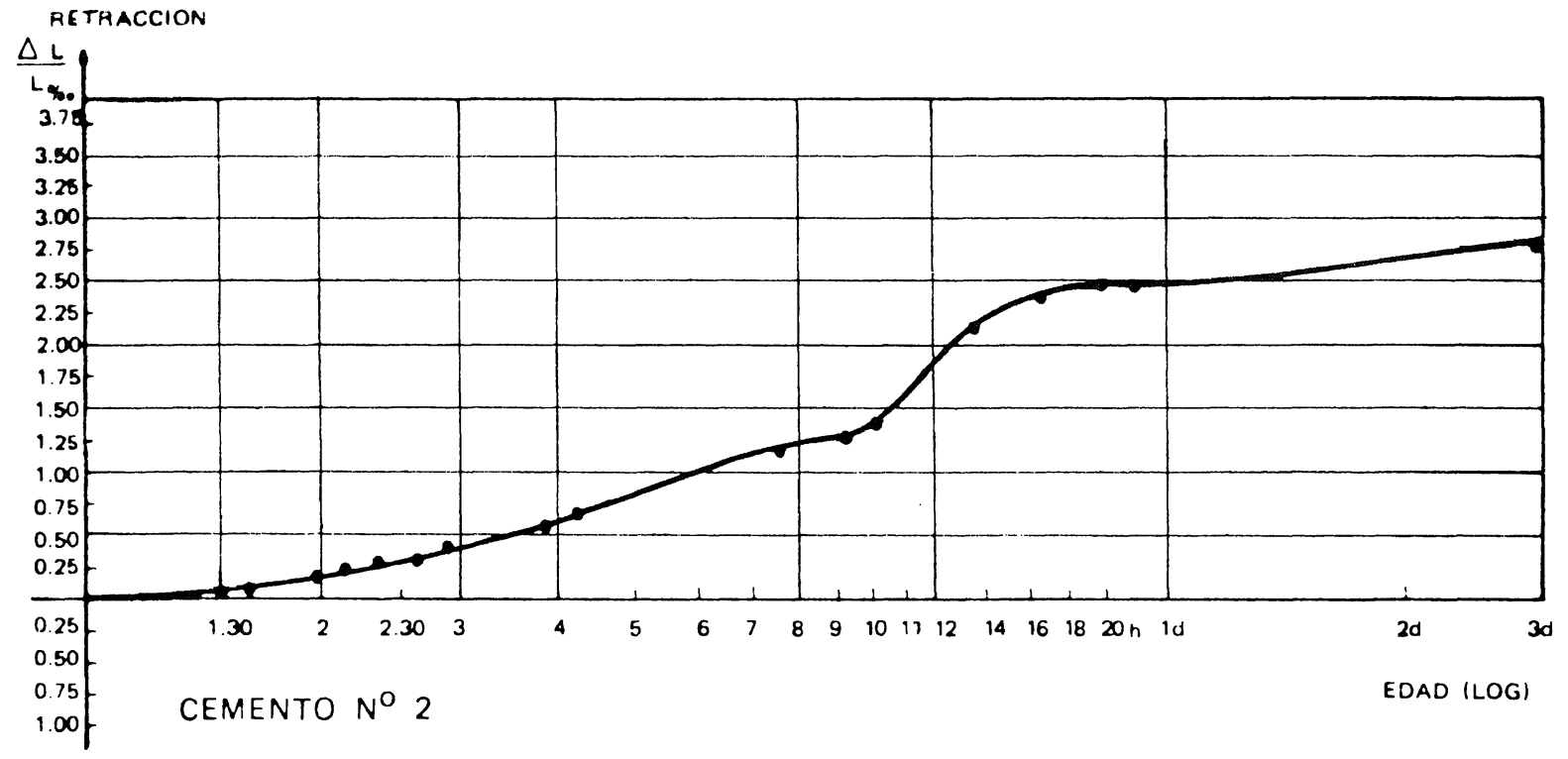

Fig. 20.-Promedio de retracciones medidas en miniprobetas de pasta del cemento $\mathrm{N} .^{\circ} 2$ en función de la edad. En este caso, es posible apreciar la inflexión que señala el comienzo de la segunda retracción. 


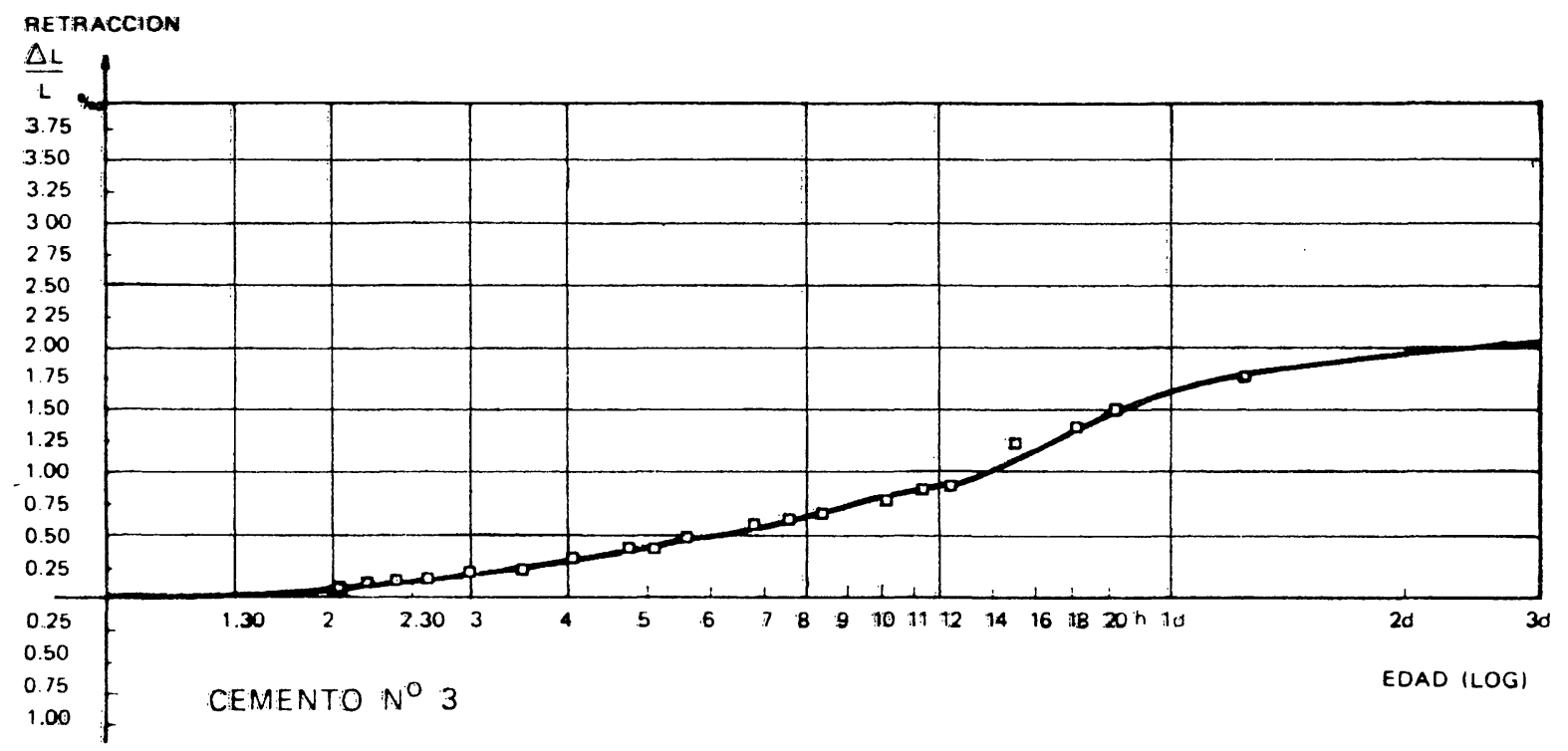

Fig. 21.-Promedio de retracciones medidas en miniprobetas de pasta del cemento $\mathrm{N}^{\circ} 3$ en función de la edad. El inicio de la segunda retracción se distingue solamente por el cambio de pendiente de la curva de ajuste.

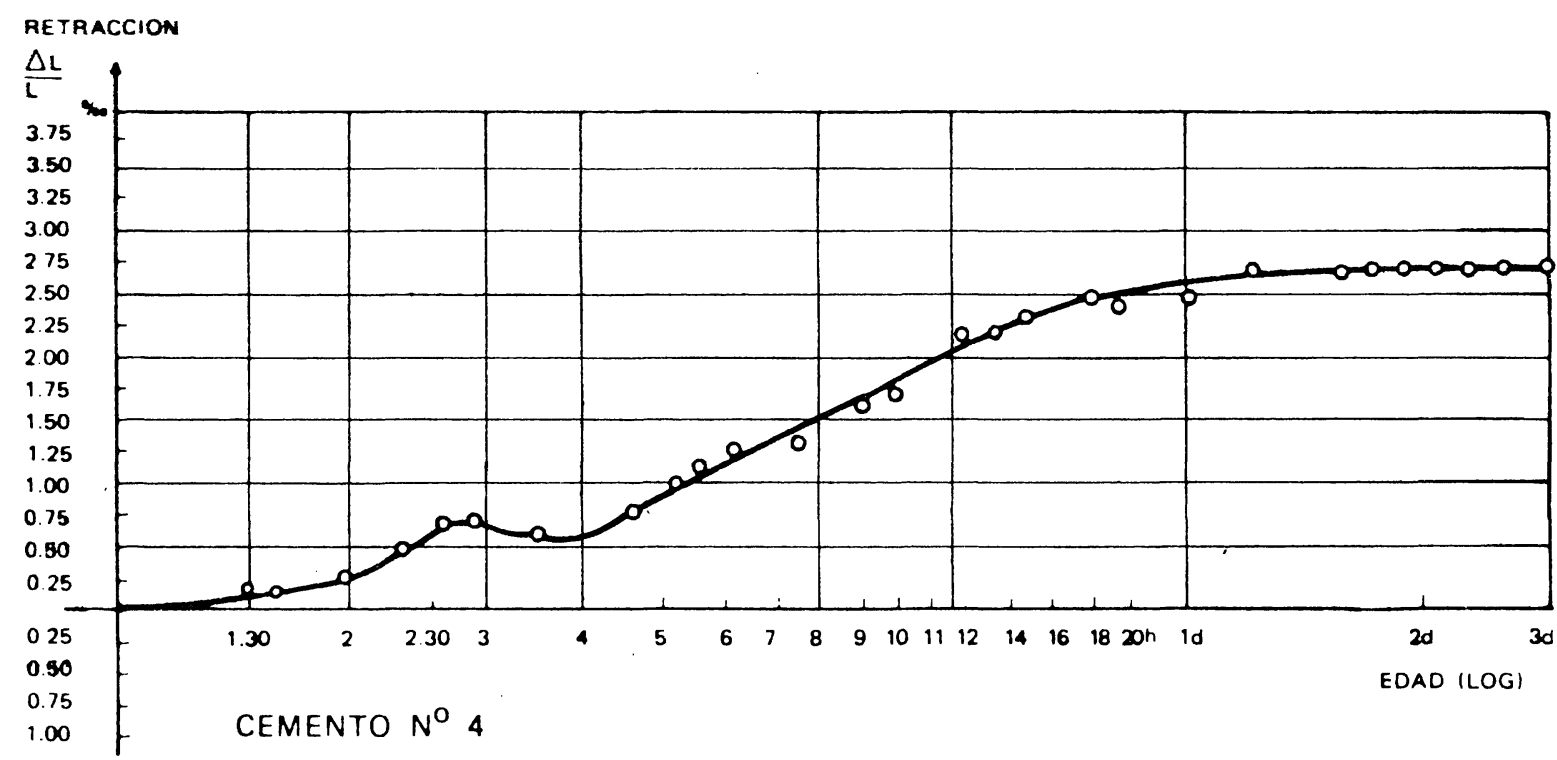

Fig. 22.-Promedio de retracciones medidas en miniprobetas de pasta del cemento $\mathrm{N}^{\circ} 4$ en función de la edad. La curva ajustada muestra las etapas de primera retracción, hinchamiento y segunda retracción en mejor forma que los casos anteriores. 


\section{ANALISIS DE LOS RESULTADOS}

El examen visual de las curvas de retracción de los cuatro cementos permiten determinar que la dispersión entre probetas, compañeras de una misma muestra, es relativamente pequeña. J. Baron (11) concluye, en un estudio experimental sobre la dispersión de la medida de retracción de pastas de cemento normal, que ella da resultados tan dispersos como los numerosos otros ensayos y medidas concernientes a los cementos. Esto significa que el método empleado en este trabajo es bastante confiable y fácilmente reproducible, a pesar de las exigencias de delicadeza y sacrificio que requiere.

En las curvas de retracción media de cada uno de los tipos de cemento, se pueden estudiar las distintas etapas de deformación que experimenta la pasta. Considerando que la confección de las probetas se realizó por un procedimiento de fuerte compactación que dejó a las pastas con una relación agua: cemento muy baja, la sedimentación aparentemente fue nula y sólo debió producirse la retracción vertical correspondiente. En esta situación, en todos los cementos se aprecia una primera retracción horizontal desde el inicio de las medidas hasta un punto determinado, según el tipo de cemento, con una pendiente ligeramente variable. El fin de la primera retracción y la etapa de hinchamiento sólo resultó apreciable en el cemento $\mathrm{N}^{\circ}{ }^{\circ}$ y cuando recién comenzaba su fraguado. Ello no es de extrañar, porque las inflexiones de las curvas de retracción, que señalan los periodos de hinchamiento y comienzo de la segunda retracción a veces pasan desapercibidas en pastas de cemento, según el método empleado, e incluso, no son visibles sobre morteros (fig. 7). De esta manera para los cementos 1,2 y 3 , se puede decir que la segunda retracción se inicia inmediatamente después de la primera, acusando su presencia sólo por un cambio de pendiente, indicadora de velocidades de retracción máximas. Ellas aparecen en la Tabla II, junto al periodo horario en que se producen y los correspondientes tiempos de fraguado.

T ABLA II

Velocidades máximas de segunda retracción (V.M.S.R.) en miniprobetas de pasta de cemento

\begin{tabular}{|c|c|c|c|c|}
\hline \multirow{2}{*}{$\begin{array}{c}\text { Cemento } \\
\mathbf{N .}^{\mathbf{0}}\end{array}$} & Inicio & Fin & $\begin{array}{c}\text { V.M.S.R. } \\
\mathbf{m m} / \mathbf{m} / \mathbf{h}\end{array}$ & \begin{tabular}{c} 
Intervalo de ocurrencia \\
\cline { 2 - 4 }
\end{tabular} \\
\cline { 2 - 4 } 1 & $3: 30$ & $4: 40$ & 0,111 & $\mathbf{E d a d}, \mathbf{h}: \mathbf{m i n}$ \\
2 & $2: 20$ & $3: 10$ & 0,149 & $5: 00-11: 00$ \\
3 & $2: 40$ & $3: 45$ & 0,113 & $12: 00-16: 00$ \\
4 & $4: 25$ & $5: 45$ & 0,155 & $4: 00-15: 00$ \\
\hline
\end{tabular}

Se puede observar que, a excepción del cemento $\mathrm{N}^{\circ}{ }^{\circ}$, las velocidades máximas de retracción se produjeron después de finalizado el tiempo de fraguado normal.

A partir del término del desarrollo de la máxima velocidad de retracción, los cuatro cementos continúan experimentando deformación a menor ritmo y alcanzando, a la edad de 3 días, los valores que se señalan en la Tabla III.

\section{T ABLA III}

Retracciones máximas a 3 días de edad (R.M.3) en miniprobetas de pastas de cemento

\begin{tabular}{|c|c|}
\hline $\begin{array}{c}\text { Cemento } \\
\mathbf{N}^{\mathbf{0}}\end{array}$ & $\begin{array}{c}\text { R.M.3 } \\
\mathbf{m m} / \mathbf{m}\left(\mathbf{~}^{\mathbf{0}} / \mathbf{o o}\right)\end{array}$ \\
\hline 1 & 1,55 \\
2 & 2,75 \\
3 & 2,05 \\
4 & 2,70 \\
\hline
\end{tabular}


Las retracciones máximas obtenidas a los tres días presentan diferencias significativas entre algunos de los cuatro cementos. El cemento $\mathrm{N} .^{\circ} 1$ muestra la menor retracción final y los cementos $\mathrm{N}$.os 2 y 4, las mayores. Como primer factor de influencia para explicar este hecho, se puede considerar la superficie específica que aparece en la Tabla I. En efecto, el cemento N. ${ }^{\circ} 1$ registró una finura de $4.000 \mathrm{~cm}^{2} / \mathrm{g}$ contra $4.200 \mathrm{~cm}^{2} / \mathrm{g}$ de los cementos N.os 2 y 4 . M. Venuat (23) encontró que el aumento de finura entre 3.000 y $4.000 \mathrm{~cm}^{2} / \mathrm{g}$ aumenta la retracción en un $30 \%$. Sin embargo, la escasa diferencia registrada no es suficiente para justificar la menor retracción del cemento $\mathrm{N}^{\circ} 1$, por lo que cabe considerar como segundo factor el menor contenido de $\mathrm{C}_{3} \mathrm{~A}$ de este cemento que, como se señala en la misma Tabla I, era de sólo 5,0 \% contra 10,0 y $8,0 \%$ de los cementos N. ${ }^{\text {ss }} 2$ y 4, respectivamente. Experiencias de diversos origenes, Kühl, R. Dittrich, W. Czernin, M. Venuat, T. C. Powers citados por Keil (24) coinciden prácticamente al señalar como coeficientes aproximados de retracción de las fases mineralógicas del clínker, los siguientes:

$$
\mathrm{C}_{3} \mathrm{~S}=0,048 ; \quad \mathrm{C}_{2} \mathrm{~S}=0,020 ; \quad \mathrm{C}_{3} \mathrm{~A}=0,102 ; \quad \mathrm{C}_{4} \mathrm{AF}=0,025
$$

Se confirma entonces la importancia del contenido de $\mathrm{C}_{3} \mathrm{~A}$ sobre el fenómeno de retracción.

Por otra parte, los dos cementos que presentan los valores más altos de retracción final a 3 días de edad, asi como las mayores velocidades de segunda retracción, contienen adiciones diferentes. El cemento $\mathrm{N}^{\circ} 2$ posee adición puzolánica semejante a la de los cementos $\mathrm{N}$. os 1 y 3 y el cemento N. ${ }^{\circ} 4$ posee una adición de escoria de alto horno. No se conocen muchos antecedentes comparativos sobre los efectos que ambos tipos de adición producen sobre el fenómeno de la retracción. En un estudio realizado por M. Ray, R. Lesage, J. Baron, A. M. Paillere, J. L. Nissoux y R. Guinez (25) se compararon las retracciones de diferentes cementos y se constató que la velocidad máxima de segunda retracción de un cemento portland de escorias (CPAL 400) era superior que la de un cemento portland puzolánico (CPAZ 400). En cualquier forma, ambos tipos de cemento mostraron velocidades máximas de retracción mucho menores que los cementos portland ensayados (CPA 500 y CPA 400), lo que señala un efecto beneficioso adicional de las adiciones.

En los factores de influencia considerados hasta aquí, el cemento $\mathrm{N}^{\circ}{ }^{\circ} 3$ mostró valores intermedios, concordantes con la velocidad máxima de la segunda retracción y con la retracción final a 3 dias de edad, mostradas en los resultados de ensayo.

En general, las retracciones finales registradas por los cuatro cementos de este estudio están dentro de los márgenes normales. A. Neville (26) cita retracciones observadas en pasta de cemento que fluctúan entre 4 y $10 \mathrm{~mm} / \mathrm{m}$ y J. Baron (11), menciona retracciones medidas en mortero entre 1 y $20 \mathrm{~mm} / \mathrm{m}$. Sin embargo, las velocidades máximas de segunda retracción se asemejan a las mayores informadas por otros investigadores, empleando otros métodos y otro tipo de probetas (fig. 23). A pesar de la bajisima relación agua : cemento final de las pastas de ensayo, que produciria una retracción menor (fig. 5), los mayores valores obtenidos se justifican por la menor dimensión de las probetas (fig. 8), por la ausencia de roce de paredes de moldes (fig. 9), y especialmente, por la aceleración del secamiento de las miniprobetas al mantener expuestas al ambiente

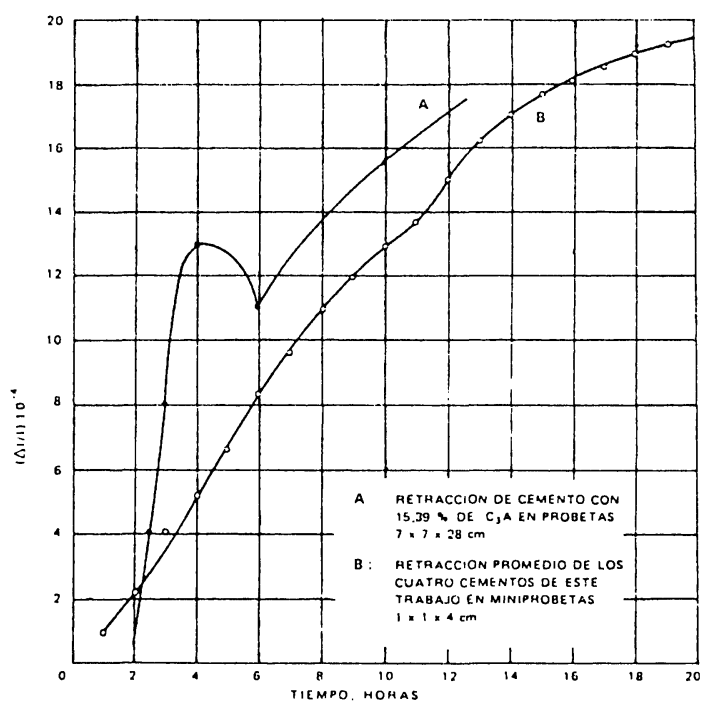

Fig. 23 
desecador, cinco de las seis caras de cada prisma. T. C. Hansen y A. H. Marrock, según F. Keil (24) han opinado ya que la retracción final disminuye cuando aumenta la relación volumen : superficie.

\section{CONCLUSION}

Los resultados obtenidos, por el método de medición de la retracción en miniprobetas de pastas de cemento, no son comparables con los provenientes de otros métodos en valores absolutos, pero permiten conocer perfectamente el comportamiento del material durante su estado plástico $\mathrm{y}$, sobre todo, el periodo en que se produce y el valor que alcanza la máxima velocidad de retracción de cada cemento. Ensayos más exhaustivos serán necesarios para confirmar los resultados obtenidos en este trabajo. En todo caso, es ya conveniente que internacionalmente se establezca y se acepte un método de ensayo simple para determinar la retracción de pastas de cemento, morteros y hormigones en su primera edad. En apariencia, hay concordancia en que para medidas de retracción deben emplearse como probetas prismas de sección cuadrada y relación longitud/arista igual a 4 . Pero se han empleado indistintamente probetas de $7 \times 7 \times 28 \mathrm{~cm}$, tanto para pasta de cemento, como para morteros. De manera similar, existen otros detalles pertenecientes a la metodologia de ensayo que requieren mayor definición para obtener valores comparativos $\mathrm{y}$, consecuentemente, relaciones válidas con otros parámetros del hormigón.

\section{R E F E R E N C I A S}

(1) LAMANA, A.: Informe sobre agrietamiento del hormigón en la variante Lanco del camino Longitudinal Sur. Certificado de Ensayo N. ${ }^{\circ} 143.032$, IDIEM, Santiago, Agosto, 1978.

(2) TYLER, R. G.: Creep, Shrinkage and elastic strain in concrete bridges in the United Kingdom, 1963-71. Magazine of Concrete Research. Vol. 28, N. ${ }^{\circ}$ 95. June 1976.

(3) SHALON, R. RAVINA, D. and JEAGERMAN, C. H.: Hot-dry climate effect on stress development in shrinkagecompensating concrete. Journal of the American Concrete Institute, March 1977. N. ${ }^{\circ}$ 3, Proceeding. Vol. 7.

(4) BROKER, F. W. y SIMATUPANG, M. H.: The effect of polythylene glycol on some physical properties of hardened portland cement paste. Zement-Kalk-Gips N. ${ }^{\circ} 2,1976$, pp. 65-70.

(5) HOBBS, D. W.: Expansion and shrinkage of over sulphated portland cement. Cement and Concrete Research and International Journal, Pergamon Press, Vol. 8, N. ${ }^{\circ}$ 2. March, 1978.

(6) BARON, J.: Mesures du premier retrait des bétons hydrauliques. Colloque International sur le Retrait des Bétons Hydrauliques, RILEM, Madrid, 1968.

(7) RAVINA, D. y SHALON, R.: Plastic shrinkage cracking. ACI, Journal, Abril 1968, Procceding 65.

(8) KLOCK, B. y SHERIDAN, R.: Study of shrinkage in concrete frames. Journal of the American Concrete Institute, Vol. 28, N. ${ }^{\circ} 8$, febrero 1957. Proceeding, Vol. 53, pp. 791-796.

(9) KRAL, S. and GEBAUER, V.: Shrinkage and cracking of concrete at early ages. Advances in Concrete Slab Technology. Ed. R. K. Dhir y J. G. L. Munday Pergamon Press, 1980.

(10) BARON, J. EL KASSEM, N., GUIEYSSE, B.: Mise au point d'un essai simplifié de second retrait. Bulletin de Liaison des Laboratoires des Ponts et Chaussées N. ${ }^{\circ} 89$, mayo-junio 1977, pp. 39-44.

(11) BARON, J.: Fissuration du bèton par hydratation localement différée du ciment. Rapport de Recherche N." 15 , Ministere de l'Equipement et du Logement, L.C.P.C. Mai, 1971.

(12) CZERNIN, W.: La química del cemento. Ed. Palestra. España 1963.

(13) JEAGERMAN, C. H. y GLUCKLICH, J.: Effect of Plastic Shrinkage on Subsequent Shrinkage and Swelling of the Hardened Concrete. Colloque International sur le Retrait des Bétons Hydrauliques, RILEM, Madrid, 1968.

(14) POWER, T. C.: Properties of fresh concrete, Wiley, 1968. 
(15) LERCH, W.: Plastic Shrinkage. Journal of the American Concrete. Vol. 28, N. ${ }^{\circ}$ 8, (febrero 1957), Proceedings. Vol. 53, pp. 797-802.

(16) FERNANDEZ, P. J. M.: Contribución al estudio de la mecánica de formación de la ettringita durante el periodo plástico de la pasta de cemento. Materiales de Construcción N. ${ }^{\circ} 147$, Instituto Eduardo Torroja, pp. 49-53, Madrid. España.

(17) L'HERMITE, R. et MAMILlAN, M.: Influence de la dimension et de la forme sur le retrait des pieces de beton. Colloque International sur le Retrait des Bètons Hydrauliques, RILEM, Madrid, 1968.

(18) LEVY. P.: La Precision des mesures de retrait hydrauliques aprés prise. Colloque International sur le Retrait des Bétons Hydrauliques. RILEM, Madrid, 1968.

(19) MAMillaN, M.: Note sur la precision des mesures de retrait. Colloque International sur le Retrait des Betons Hydrauliques. RILEM, Madrid, 1968.

(20) TAYLOR, R. y FRODIN, J. A.: Contribution the design of composite beams to satisfy deflexion criteria. The Institution of Civil Emgineering. Proceedings, Aarch, 1977. Vol. 63. Part. 63. Part 2, pp. 105-122.

(21) PARROT, L. J.: Recoperable and intrecoperable deformation of theatcured! cement praste. Magazine of Concrete Research. Vol. 29. N.. 98, March i1977.

(22) HOBBS, D. W.:- Influence of specimen geometry upon weight change and shrinkage of air-dried concrete specimens. Magazine of Concrete Researdh. Vol. 29, N. ${ }^{\circ}$ 99, June 1977.

(23) PAPADAKIS, M. y VENUAT, M.: Fabricación, caracteristicas y aplicaciones de los diversos tipos de cemento. Editores Técnicos Asociadøs, S. A., Barcelona, España, 1968.

(24) KEIL, F.: Cemento. Fabricación. Propiedades. Aplicaciones. Editores Técnicos Asociados, S. A., Barcelona, España, 1973.

(25) RAY, M., LESAGE, R., BARON, J., PAILlere, A. M., NISSOUX, J. L., GUINEZ, R.: Les ciments pour bétons routieres. Recherche des spécifications optimales. Chaussées en béton. Bulletin de Liaison des Laboratoires des Ponts et Chaussées, Septembre, 1978.

(26) NEVILLE, A. M.: Properties of Concrete. Ed. Pitman, Londres, 1981.

\section{publicacion del i.e.t.c.c.}

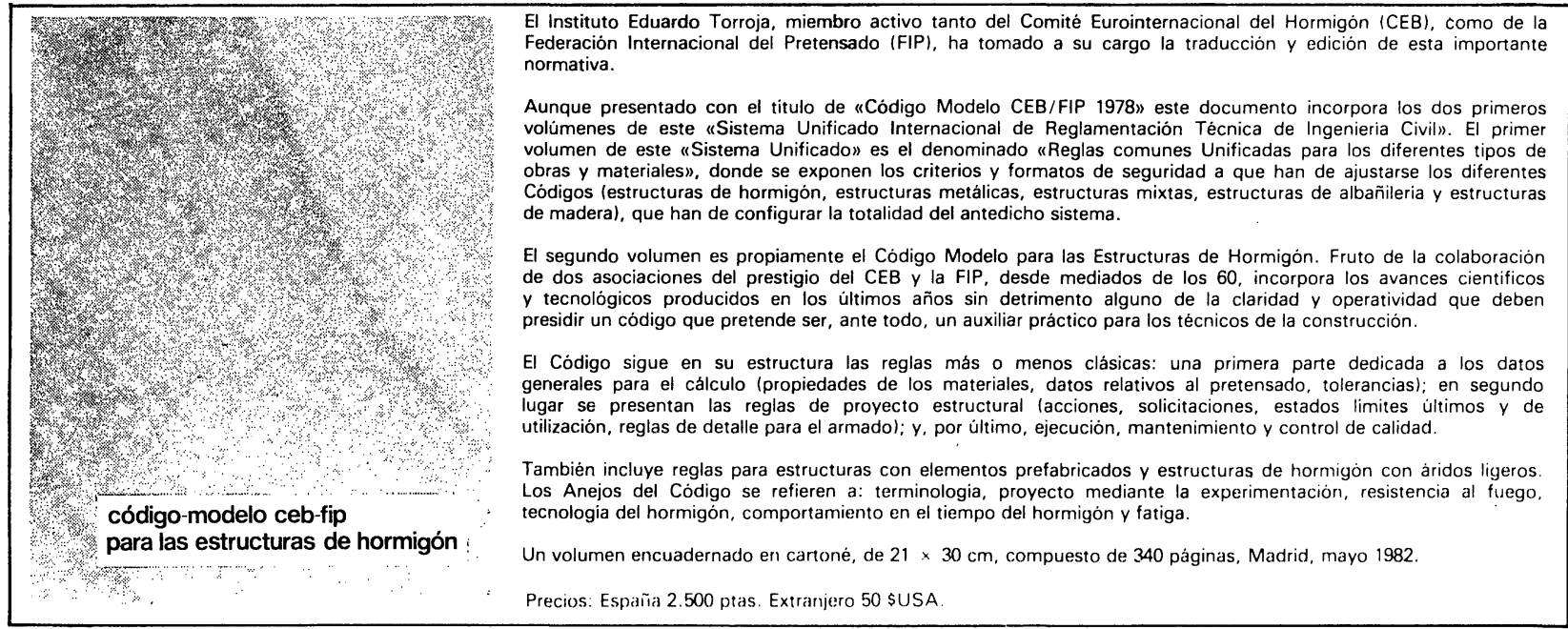

